\title{
UNCERTAINTY QUANTIFICATION IN ULTRASONIC GUIDED-WAVES BASED DAMAGE LOCALIZATION
}

\author{
Sergio Cantero-Chinchilla ${ }^{1,4}$, Juan Chiachío ${ }^{2}$, Manuel Chiachío ${ }^{3}$, Dimitrios \\ Chronopoulos $^{1}$, Arthur Jones ${ }^{1}$, Yasser Essa ${ }^{4}$, Federico Martín de la Escalera ${ }^{4}$ \\ ${ }^{1}$ Institute for Aerospace Technology \& The Composites Group, The University of Nottingham \\ Nottingham, UK \\ e-mail: \{Sergio.CanteroChinchilla1, Dimitrios.Chronopoulos, Arthur.Jones\}@ nottingham.ac.uk \\ ${ }^{2}$ Department of Naval Architecture, Ocean \& Marine Engineering, University of Strathclyde \\ Glasgow, UK \\ e-mail: juan.chiachio-ruano@strath.ac.uk \\ ${ }^{3}$ Dept. Structural Mechanics \& Hydraulics Engineering, University of Granada \\ Granada, Spain \\ e-mail: mchiachio@ugr.es \\ ${ }^{4}$ Aernnova Engineering Division \\ Madrid, Spain \\ e-mail: \{yasser.essa, federico.martindelaescalera\}@ aernnova.com
}

Keywords: Bayesian inverse problem, SHM, ultrasound, guided waves, damage localization, hyper-robust model, uncertainty quantification.

\begin{abstract}
Bayesian methods for inverse problems offer higher robustness to noise and uncertainty than deterministic, yet accurate, inference methods. Both types of techniques typically focus on finding optimal model parameters that minimize an objective function, which compares model output with some acquired data. However, uncertainties coming from different sources, such as: (1) the material manufacturing process, (2) material's mechanical properties, (3) measurement errors, or (4) the model and its parameters, may cause inference errors and loss of information should they are not properly taken into account. These uncertainties might have important safety and economic consequences in damage-related applications, such as in structural health monitoring of aerospace structures. This paper aims at illustrating the benefits of using probability based methods instead of deterministic approaches. A case study is presented, which illustrates the use of a hyper-robust Bayesian damage localization method when compared to a deterministic one. The results show that Bayesian inverse problem is more robust to data noise and uncertainties stemming from the model parameters than deterministic methods.
\end{abstract}




\section{INTRODUCTION}

Damage localization and reconstruction in plate-like structures using guided-wave based structural health monitoring (SHM) have been mainly addressed using post-processing techniques applied to ultrasonic signals [1]. The exploration of large areas with a small attenuation [2] is one of the most remarkable characteristics that has led industries, such as the aerospace industry, to focus on guided-waves. Sparse or phased-array sensors' layouts are typically placed so that the structure is actively interrogated on demand, which confers higher accuracy and reliability [3]. Potential safety and economical implications in condition-based maintenance are extra-motivations for the use of this SHM technique. The required damagerelated information is extracted from the data by using inverse problems (IPs) that minimize the distance between model predictions and observations [4].

IPs can be solved using different methods such as the deterministic or the Bayesian ones. Deterministic approaches seek the best value of the model parameters that provides the closest prediction to the available observation. Alternatively, Bayesian inverse problems (BIPs) provide a set of parameters values associated to their posterior plausibilities, namely the posterior probability density function (PDF). To this end, the prior degree of belief of the parameters is updated by using observations (data) and the Bayes' Theorem [5, 6]. In the particular case of ultrasoundbased damage detection, two general approaches are typically adopted: (1) model-based IPs, whereby detailed damage information (e.g. the severity of damage as residual strength) [7] can be obtained from the measured signal at a considerable computational cost; and (2) efficient IPs based on signal features, whereby other relevant information, e.g. the damage position or severity, can be obtained [2, 8-10]. Among them, the time-of-flight (ToF) has been extensively used as a signal feature for its efficiency in obtaining information about material properties along with damage localization using post-processing scattered signals. It is worth mentioning that ultrasound-based damage localization conveys sources of uncertainty that are mostly related to the measurement system and physical properties of the material.

To partially address this modeling issue, a number of researchers have proposed the use of BIP applied to ultrasound based damage localization [11, 12]. More recently, a Bayesian framework, which combines the information coming from different post-processing techniques, i.e., time-frequency models [13-16], using their posterior plausibilities to obtain a more robust damage localization, has been proposed in [17]. In this paper, a comparison between BIPs and deterministic IPs in the context of ultrasonic guided-wave based damage localization is provided. In the context of BIP, probability is interpreted as a multi-valued logic that expresses the degree of belief of a proposition conditioned on the given information [5, 18]. Both deterministic and Bayesian approaches are applied to an aluminum plate with one and two damaged areas. The asymptotic independent Markov sampling (AIMS) [19, 20] algorithm is adopted to solve the resulting BIP, while genetic algorithms (GA) are used to solve the deterministic IP. In general, the results show that BIP is superior in terms of robustness of the damage reconstruction while the deterministic problem provide very efficient solutions but at risk of obtaining incomplete and biased information.

The remainder of the paper is organized as follows: Section 2 comprises the probabilistic methodology used to obtain the robust estimate of the ToF for each sensor. In Section 3 , the proposed framework is applied in two case studies and the results are discussed. Finally, Section 4 provides concluding remarks. 


\section{METHODOLOGY}

\subsection{Bayesian damage localization}

In this section, the ultrasonic guided-wave based damage localization is addressed by using a model-based BIP using an ellipse-based ToF model [21]. To this end, $N_{p}$ actuator-sensor paths are considered in a plate-like structure to excite and receive guided-waves for damage detection by screening changes of their ToF. To this end, the ToF information of the scattered signals can be theoretically obtained as follows [22]:

$$
\operatorname{ToF}^{(a-s)}=\frac{\sqrt{\left(X_{d}-X_{a}\right)^{2}+\left(Y_{d}-Y_{a}\right)^{2}}}{V_{a-d}}+\frac{\sqrt{\left(X_{d}-X_{s}\right)^{2}+\left(Y_{d}-Y_{s}\right)^{2}}}{V_{d-s}}
$$

where $\left(X_{d}, Y_{d}\right)$ are the coordinates of the damage, $\left(X_{a}, Y_{a}\right)$ are the actuator transducer coordinates, $\left(X_{s}, Y_{s}\right)$ are the coordinates of one arbitrary sensor transducer, and $V_{a-d}$ and $V_{d-s}$ are the wave propagation velocities of the actuator-damage and damage-sensor paths respectively. These velocities are the same under the assumption of isotropic materials and a concentrated damage within a bounded region, i.e. $V=V_{a-d}=V_{d-s}$.

To probabilistically describe the ToF model given by Equation (1), a set of uncertain model parameters $\mathbf{m}=\left\{X_{d}, Y_{d}, V\right\}$ are considered in this problem to describe the uncertainty about the damage coordinates as well as the wave propagation velocity. The set $\mathbf{m}$ of model parameters is augmented with a model error term $e \in \mathbb{R}$, resulting in a set of model parameters defined as $\boldsymbol{\theta}=\left\{\mathbf{m}, \sigma_{e}\right\}=\left\{X_{d}, Y_{d}, V, \sigma_{e}\right\} \in \boldsymbol{\Theta}$, where $\sigma_{e}$ is the standard deviation of the error term $e$ and $\Theta$ is the model parameter space. The referred model error term $e \in \mathbb{R}$ is considered to account for the non-existence of a theoretical ToF model that fully represent the reality, so that [17]:

$$
\operatorname{ToF}_{\mathcal{D}}^{(a-s)}=\operatorname{ToF}_{M}^{(a-s)}(\mathbf{m})+e=\operatorname{ToF}_{M}^{(a-s)}(\boldsymbol{\theta})
$$

where subscripts $M$ and $\mathcal{D}$ from $\operatorname{ToF}_{M}^{(a-s)}$ and $\operatorname{ToF}_{\mathcal{D}}^{(a-s)}$ refer to modeled and measured ToF, respectively. Note in Equation (2) that $e$ provides the discrepancy between $\operatorname{ToF}_{M}^{(a-s)}$ and $\operatorname{ToF}_{\mathcal{D}}^{(a-s)}$ values. By the PMIE, this error term can be conservatively described as a zero-mean Gaussian distribution with covariance $\sigma_{e}$ as $\mathcal{N}\left(0, \sigma_{e}\right)$.

Next, the posterior PDF of the model parameters given the ToF data $\mathbf{D}=\left\{D^{(1)}, \ldots, D^{(N)}\right\}$, where $N$ is the total number of active sensors, by applying the well-known Bayes' Theorem as:

$$
p(\boldsymbol{\theta} \mid \mathbf{D})=\frac{p(\mathbf{D} \mid \boldsymbol{\theta}) p(\boldsymbol{\theta})}{p(\mathbf{D})}
$$

where $p(\boldsymbol{\theta})$ is the prior PDF of the model parameters, and $p(\mathbf{D} \mid \boldsymbol{\theta})$ is the likelihood function for the set of data $\mathbf{D}$. Given the stochastic independence of the measurements, the likelihood can be expressed as $p(\mathbf{D} \mid \boldsymbol{\theta})=\prod_{k=1}^{N} p\left(D^{(k)} \mid \boldsymbol{\theta}\right)$. Finally, $p(\mathbf{D})$ is the evidence of the data under the model specified by $\boldsymbol{\theta}$. This term, which acts as a normalizing factor within the Bayes' theorem, can be bypassed through sampling, e.g. using Markov Chain Monte Carlo (MCMC) methods [23]. In this paper, the AIMS algorithm [19, 20] is used as MCMC method due to its efficiency in addressing multimodality of the posterior PDF. Thus, Equation (3) can be rewritten as:

$$
p(\boldsymbol{\theta} \mid \mathbf{D}) \propto\left\{\prod_{k=1}^{N} p\left(D^{(k)} \mid \boldsymbol{\theta}\right)\right\} p(\boldsymbol{\theta})
$$




\subsection{Deterministic damage localization}

Similarly, the deterministic approach pursues the minimization of the discrepancy between predictions and observations. In this case, however, the error term that measures the aforementioned distance $r$ is considered as a deterministic variable as follows:

$$
\operatorname{ToF}_{\mathcal{D}}^{(a-s)}=\operatorname{ToF}_{M}^{(a-s)}(\mathbf{m})+r
$$

where $\mathbf{m}$ denotes the unknown parameters. The minimization problem can be formulated using the $L_{2}$-norm between prediction and observation, as follows:

$$
\mathbf{m}_{\text {opt }}=\arg \min _{\mathbf{m}}\left\|\mathrm{ToF}_{\mathbf{D}}^{(a-s)}-\mathrm{ToF}_{M}^{(a-s)}\right\|_{2}^{2}
$$

where $\mathbf{m}_{\text {opt }}$ is the set of optimal parameters that minimize the discrepancy between model and data. In addition, the logarithm of the objective function is considered to boost the convergence of the minimization problem, as follows:

$$
\mathbf{m}_{\text {opt }}=\arg \min _{\mathbf{m}} \log \left(\left\|\mathrm{ToF}_{\mathbf{D}}^{(a-s)}-\operatorname{ToF}_{M}^{(a-s)}\right\|_{2}^{2}+\Delta\right)
$$

where $\Delta$ is a small value that avoids the trivial solution when the objective functions tends to zero [24]. Equation (6) is then addressed by using GA [25], given that the objective function is non-convex, and hence more than one local minimum may be found. The GA are configured so that the convergence of the objective function is achieved.

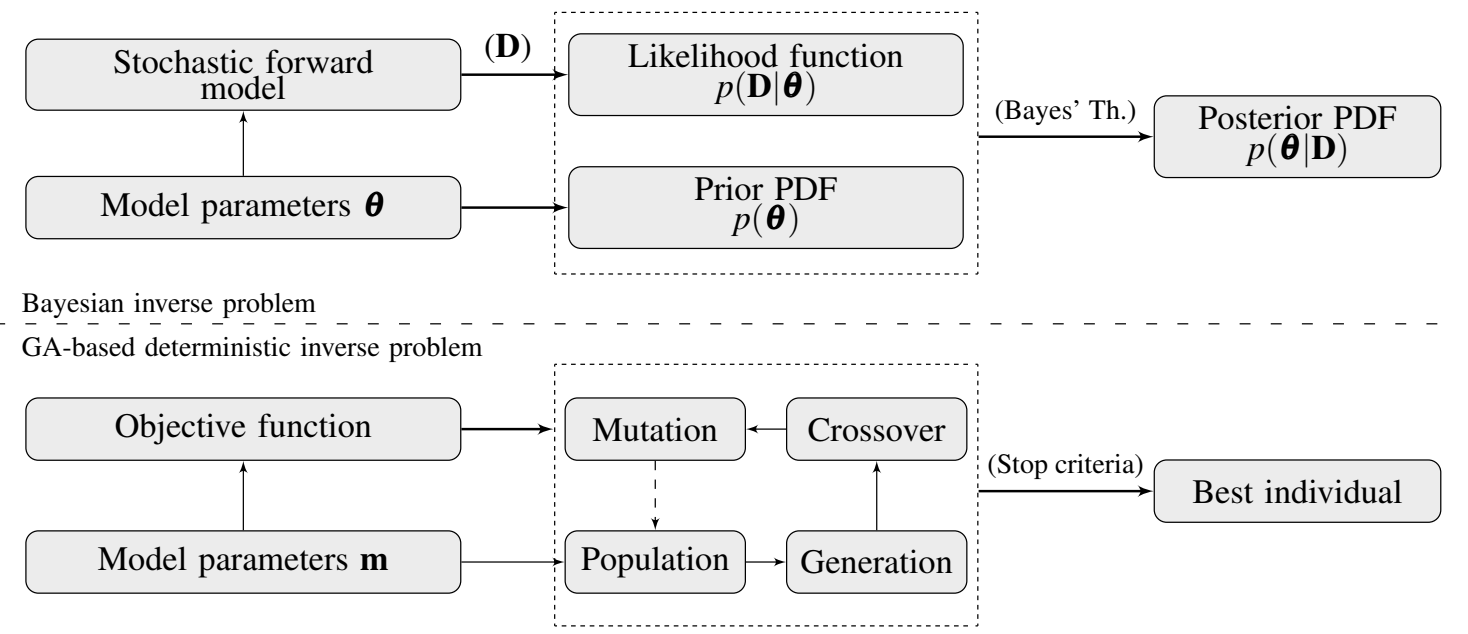

Figure 1: General framework used to compare deterministic and Bayesian IPs.

Figure 1 depicts the workflow for the comparison of both the BIP and the deterministic IP using a GA-based approach. Note that both IPs use the same ToF data to address the damage localization so that the outputs are comparable. Note also that the BIP provides the values of the model parameters associated to their posterior probabilities by the posterior PDF, while the deterministic approach only provides the best individual that minimizes the corresponding objective function (recall Eq. (7)). Thus, the BIP is able to quantify the uncertainty associated to the model parameters stemming from different sources. 


\section{CASE STUDIES}

\subsection{Synthetic signal generation}

For the purpose of signal generation, a $300 \mathrm{~mm} \times 300 \mathrm{~mm}$ aluminium plate of $2 \mathrm{~mm}$ thickness has been modeled in Abaqus. The material is considered to be an aluminum alloy 2024-T351 with Young's modulus $E=73.1 \mathrm{GPa}$, density $\rho=2780 \mathrm{~kg} / \mathrm{m}^{3}$, and a Poisson's ratio of 0.33 . Here, the damage is modeled as a rectangular hole of dimensions $2 \mathrm{~mm} \times 4 \mathrm{~mm}$. S4R (4-node, quadrilateral, stress-displacement elements with reduced integration and large-strain formulation) shell elements [26] are used with $1 \mathrm{~mm}$ mesh size to avoid spatial aliasing. The excitation is modeled as a perpendicular action with a 5 cycle sine tone-burst centered at $f=100 \mathrm{kHz}$. In addition, the sensors are arbitrarily placed as can be observed in Figure 2.

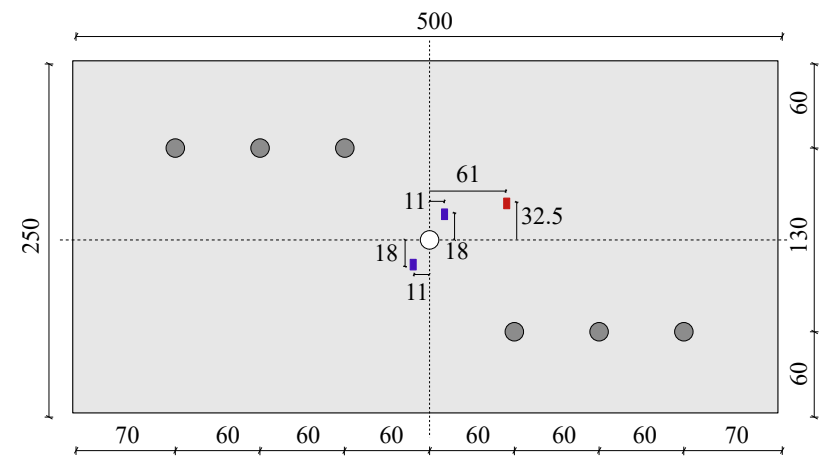

Figure 2: Layout of the aluminum plate with both one and two damaged areas. The red area correspond to a single damage location in case study 1 , while the two blue damaged areas correspond to the case study 2.

The acquired signals are then post-processed by applying different time-frequency models, which are then ranked based on their posterior plausibilities. Consequently, a robust timefrequency model is built by using such probabilities [17]. The robust ToF values are then used as data to reconstruct the damage location by using the ToF model-based approaches, i.e., the deterministic and the Bayesian ones.

\subsection{One damaged area}

Figure 3 a depicts the damage reconstruction when one damaged area is simulated. In this case study, both the BIP, whose posterior PDF is respresented by iso-probability lines, and the deterministic IP, whose best individual is depicted by a gray point, provide similar damage localization. Here, the deterministic IP stands out computationally due to its efficiency in providing the minimum. However, the superior robustness of the posterior PDF provided by the Bayesian approach, which accounts for uncertainties from several sources, is manifested in its dispersion, while the deterministic solution provides one unique value. As can be observed in Figure 3a, the deterministic solution is located at the tail of the postetrior PDF, which is the result of a different wave propagation velocity inference. In particular, the velocity obtained by the deterministic approach is $1962 \mathrm{~m} / \mathrm{s}$, while the mean of the marginal posterior PDF obtained by the BIP is $2000.4 \mathrm{~m} / \mathrm{s}$. This small variation is the cause of the misplacement of the damage position in the deterministic IP with respect to the BIP. These results emphasize the strengths of BIP when dealing with uncertain data, models and parameters. 


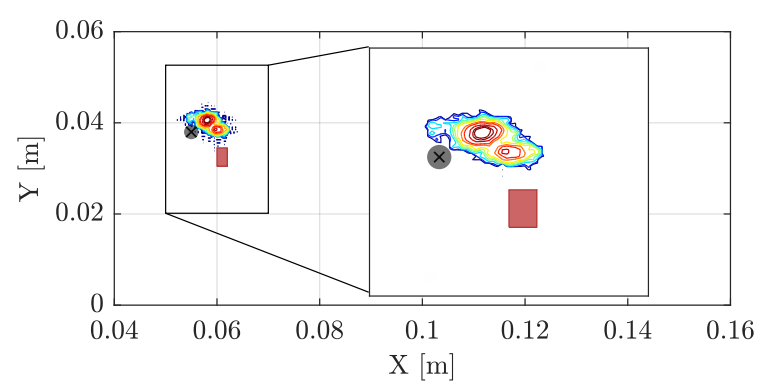

(a) One damaged area.

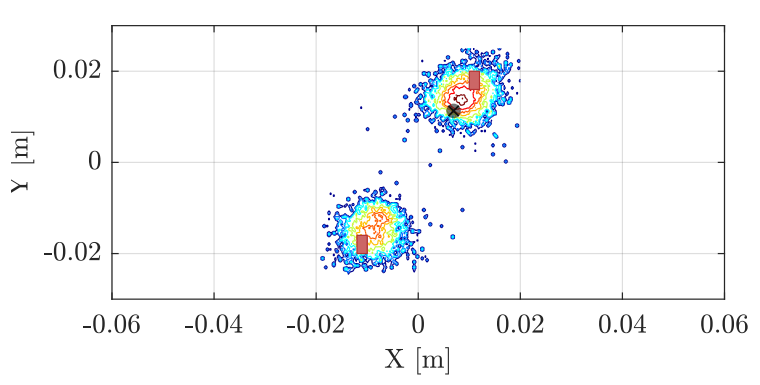

(b) Two damaged areas.

Figure 3: Comparison between the deterministic IP and BIP considering both one and two damaged areas in panels (a) and (b), respectively.

\subsection{Two damaged areas}

In case of two or more damaged areas, the BIP shows its clear superiority in comparison with the deterministic IP. As can be observed in Figure 3b, the deterministic solution of the damage location, depicted by the gray point, is relatively accurate with respect to one damaged area. This result points out one of the limitation of the GA, which is only able to provide with one minimum that corresponds to the damage location of one hole. In contrast, the Bayesian approach is able to provide with a relatively accurate damage location of both holes due to the ability of the AIMS algorithm to address multimodality. Note that the posterior PDF is depicted by iso-probability lines in Figure $3 \mathrm{~b}$. It is also worth mentioning that both the high probability areas coincide with the closer corner holes to the actuator located at the center of the plate. This behavior agrees with the wave scattering phenomenon when the guided-waves reach the square holes.

\section{CONCLUSIONS}

A comprehensive comparison between deterministic and Bayesian approaches to address ultrasonic guided-wave based damage localization has been illustrated in this paper. To this end, a state-of-the-art robust Bayesian damage localization approach that uses the AIMS as MCMC algorithm to draw samples from the posterior PDF has been compared with the classical approach of GA-based IP. Two case studies have been selected to illustrate the strengths of each approach, using one structure with one and two damaged areas, respectively. The results show the superiority of the BIP in comparison with the deterministic IP in more complex cases such as in the presence of multiple damage areas, while the GA-based approach stands out due to its efficiency in providing local minimum.

\section{ACKNOWLEDGEMENTS}

This paper is part of the SAFE-FLY project that has received funding from the European Union's Horizon 2020 research and innovation programme under the Marie Sklodowska-Curie grant agreement No 721455. In addition, the authors are grateful for the access to the University of Nottingham High Performance Computing Facility and to the University of Granada for "ROBIN" grant [30.BF.66.11.01], which partially provides support to this work. 


\section{References}

[1] J. Achenbach. Wave Propagation in Elastic Solids. North-Holland Publishing Company/American Elsevier, 1973.

[2] Z. Su, L. Ye, and Y. Lu. Guided Lamb waves for identification of damage in composite structures: A review. Journal of sound and vibration, 295(3-5):753-780, 2006.

[3] L. Wang and F. Yuan. Active damage localization technique based on energy propagation of Lamb waves. Smart Structures and Systems, 3(2):201-217, 2007.

[4] A. Tarantola. Inverse problem theory and methods for model parameter estimation, volume 89. siam, 2005.

[5] J. L. Beck. Bayesian system identification based on probability logic. Structural Control and Health Monitoring, 17(7):825-847, 2010.

[6] G. Rus, J. Chiachío, and M. Chiachío. Logical inference for inverse problems. Inverse Problems in Science and Engineering, 24(3):448-464, 2016.

[7] J. Chiachío, N. Bochud, M. Chiachío, S. Cantero, and G. Rus. A multilevel Bayesian method for ultrasound-based damage identification in composite laminates. Mechanical Systems and Signal Processing, 88:462-477, 2017.

[8] C. H. Wang, J. T. Rose, and F.-K. Chang. A synthetic time-reversal imaging method for structural health monitoring. Smart materials and structures, 13(2):415, 2004.

[9] J. E. Michaels and T. E. Michaels. Guided wave signal processing and image fusion for in situ damage localization in plates. Wave motion, 44(6):482-492, 2007.

[10] J. E. Michaels, A. J. Croxford, and P. D. Wilcox. Imaging algorithms for locating damage via in situ ultrasonic sensors. In Sensors Applications Symposium, 2008. SAS 2008. IEEE, pages 63-67. IEEE, 2008.

[11] G. Yan. A Bayesian approach for damage localization in plate-like structures using Lamb waves. Smart Materials and Structures, 22(3):035012, 2013.

[12] C. Fendzi, N. Mechbal, M. Rebillat, M. Guskov, and G. Coffignal. A general Bayesian framework for ellipse-based and hyperbola-based damage localization in anisotropic composite plates. Journal of Intelligent Material Systems and Structures, 27(3):350-374, 2016.

[13] L. Cohen. Time-frequency Analysis: Theory and Applications. Prentice-Hall, Inc., Upper Saddle River, NJ, USA, 1995. ISBN 0-13-594532-1.

[14] N. E. Huang, Z. Shen, S. R. Long, M. C. Wu, H. H. Shih, Q. Zheng, N.-C. Yen, C. C. Tung, and H. H. Liu. The empirical mode decomposition and the Hilbert spectrum for nonlinear and non-stationary time series analysis. In Proceedings of the Royal Society of London A: mathematical, physical and engineering sciences, volume 454, pages 903-995. The Royal Society, 1998.

[15] C. K. Chui. An Introduction to Wavelets. Academic Press Professional, Inc., San Diego, CA, USA, 1992. ISBN 0-12-174584-8. 
[16] C. Bao, H. Hao, Z.-X. Li, and X. Zhu. Time-varying system identification using a newly improved HHT algorithm. Computers \& Structures, 87(23-24):1611-1623, 2009.

[17] S. Cantero-Chinchilla, J. Chiachío, M. Chiachío, D. Chronopoulos, and A. Jones. A robust Bayesian methodology for damage localization in plate-like structures using ultrasonic guided-waves. Mechanical Systems and Signal Processing, 122:192-205, 2019.

[18] E. T. Jaynes. Information theory and statistical mechanics. Physical review, 106(4):620, 1957.

[19] J. L. Beck and K. M. Zuev. Asymptotically independent Markov sampling: a new Markov chain Monte Carlo scheme for Bayesian inference. International Journal for Uncertainty Quantification, 3(5), 2013.

[20] K. M. Zuev and J. L. Beck. Global optimization using the asymptotically independent Markov sampling method. Computers \& Structures, 126:107-119, 2013.

[21] E. B. Flynn, M. D. Todd, P. D. Wilcox, B. W. Drinkwater, and A. J. Croxford. Maximumlikelihood estimation of damage location in guided-wave structural health monitoring. In Proceedings of the Royal Society of London A: Mathematical, Physical and Engineering Sciences, volume 467, pages 2575-2596. The Royal Society, 2011.

[22] J.-B. Ihn and F.-K. Chang. Pitch-catch active sensing methods in structural health monitoring for aircraft structures. Structural Health Monitoring, 7(1):5-19, 2008.

[23] F. Liang, C. Liu, and J. Chuanhai. Advanced Markov Chain Monte Carlo Methods. Wiley Online Library, 2010.

[24] N. Bochud. Signal processing-based identification of pathology using ultrasonics. $\mathrm{PhD}$ thesis, Universidad de Granada, 2014.

[25] M. Gen and R. Cheng. Genetic algorithms and engineering optimization, volume 7. John Wiley \& Sons, 2000.

[26] ABAQUS. Abaqus Documentation. Dassault Systèmes, Providence, RI, USA, 2016. 